\title{
Electrocardiographic features, mapping and ablation of idiopathic outflow tract ventricular arrhythmias
}

\author{
Carlo Lavalle ${ }^{1}$ (1) $\cdot$ Marco V. Mariani $^{2} \cdot$ Agostino Piro $^{2} \cdot$ Martina Straito $^{2} \cdot$ Paolo Severino $^{2} \cdot$ Domenico G. Della Rocca $^{3} \cdot$ \\ Giovanni B. Forleo ${ }^{4} \cdot$ Jorge Romero $^{5} \cdot$ Luigi Di Biase $^{5} \cdot$ Francesco Fedele $^{2}$
}

Received: 2 July 2019 / Accepted: 27 August 2019

(C) Springer Science+Business Media, LLC, part of Springer Nature 2019

\begin{abstract}
Purpose Idiopathic outflow tract ventricular arrhythmias are ventricular tachycardias or premature ventricular contractions presumably not related to myocardial scar or disorders of ion channels. These arrhythmias have focal origin and display characteristic electrocardiographic features. The purpose of this article is to review the state of the art of diagnosis and treatment of idiopathic outflow tract ventricular arrhythmias.

Methods We systematically reviewed scientific literature about idiopathic outflow tract ventricular arrhythmias selecting the most relevant papers on this topic.

Results The right ventricle outflow tract is the most common site of origin for outflow tract ventricular arrhythmias, but also left ventricle outflow tract can harbour these arrhythmias. Outflow tract ventricular arrhythmias are generally benign and may require treatment if they are symptomatic, incessant or give rise to cardiomyopathy. Radiofrequency catheter ablation is an effective and safe therapeutic strategy. A successful procedure requires a thorough preoperative analysis of the 12-lead electrocardiogram of the spontaneous arrhythmia combined with a detailed electroanatomical mapping and intracardiac echocardiography.

Conclusions Idiopathic outflow tract arrhythmias are frequent in daily clinical practice and can be successfully eliminated through discrete radiofrequency catheter ablation with low rates of complications.
\end{abstract}

Keywords Idiopathic ventricular arrhythmias $\cdot$ Ventricular outflow tract $\cdot$ Catheter ablation $\cdot$ Electrocardiogram

\section{Abbreviations \\ OT outflow tract \\ VA ventricular arrhythmia \\ RVOT right ventricle outflow tract \\ LVOT left ventricle outflow tract}

Carlo Lavalle and Marco V. Mariani contributed equally to this work.

Carlo Lavalle

carlo.lavalle@uniroma1.it

1 Department of Cardiovascular Disease, Policlinico Umberto I Hospital, Viale del Policlinico 155, 00161 Rome, Italy

2 Department of Cardiovascular, Respiratory, Nephrology, Anaesthesiology and Geriatric Sciences, "Sapienza" University of Rome, Rome, Italy

3 Texas Cardiac Arrhythmia Institute, St. David's Medical Center, Austin, TX, USA

4 Department of Cardiology, Luigi Sacco Hospital, Milan, Italy

5 Department of Cardiology, Montefiore Medical Center, New York, NY, USA
RFCA radiofrequency catheter ablation

PVC premature ventricular contraction

LBBB left bundle branch block

RBBB right bundle branch block

ECG electrocardiogram

\section{Introduction}

Idiopathic outflow tract ventricular arrhythmias (IOT-VAs) are monomorphic premature ventricular contractions (PVCs), non-sustained ventricular tachycardias (VT) or sustained monomorphic VT [1] presumably not related to myocardial scar or ion channel disorders. The right ventricular outflow tract (RVOT) is the most common site of origin (80\%), with $20 \%$ of cases arising from left ventricular outflow tract (LVOT) $[2,3]$. However, idiopathic VAs can arise from papillary muscles [4, 5], moderator band [6], atrio-ventricular valve annuli or His-Purkinje conduction system [7]. IOTVAs have a focal origin due to trigger activity and are 
amenable of discrete ablation. Although the arrhythmogenic focus is often endocardial, both intramural and epicardial sites of origin are described. Usually, IOT-VAs have left bundle branch block (LBBB) configuration with inferior axis. IOTVAs are considered benign ventricular arrhythmias although serious adverse sequelae are described, such as cardiomyopathy, impaired quality of life, incessant VT and sudden cardiac death. Usually, these VAs are asymptomatic, but, sometimes, patients report palpitations, chest pain, presyncope and, rarely, syncope. These symptoms are generally treated with medical therapy, but catheter ablation is accepted as a highly successful therapy [8].

In the complex OT region, the anatomic contiguity of structures that can potentially harbour VAs makes the identification of the site of origin (SOO) challenging, though ECG usually displays peculiar features useful to predict the origin of VAs before ablation. Preoperative ECG analysis, electroanatomic mapping and intracardiac echocardiography (ICE) are fundamental to increase ablation success rate and to minimize complications and procedural time.

\section{Anatomic considerations and electrocardiographic features of IOT-VAs}

Outflow tracts include the RVOT and LVOT. In particular, the RVOT is an infundibular extension of the ventricular cavity which connects to the pulmonary artery; the inflow and outflow are separated by the supraventricular crest. The pulmonary root is the distal part of RVOT and supports the 3 semilunar leaflets of the pulmonic valve (PV). The PV is located 1$2 \mathrm{~cm}$ superior from the aortic valve and attaches at the sinotubular junction. When viewed from a transverse superior perspective, the pulmonary anterior cusp is actually anterior and leftward, the pulmonary right cusp is anterior and rightward and the pulmonary left cusp is posterior. The main body of RVOT courses anterior to the LVOT and in its superior aspects it extends leftward of the LVOT and the aortic annulus. The LVOT, which connects to the aorta, is nearly indistinguishable from the rest of the ventricle, and the anterior leaflet of the mitral valve is responsible for separating the inflow and the outflow. The aortic root is composed of the sinuses of Valsalva, the valve leaflet or cusps (left, right and noncoronary cusp) and the interleaflet triangles. The superior margin of aortic root is the sinotubular junction, and the inferior margin is the attachment of the semilunar cusps. At the base of the right and left sinuses of Valsalva, there usually are sleeves of ventricular myocardium because the base of the aortic cusps attaches below the ventriculoarterial junction, which is the demarcation between ventricular myocardium and fibroelastic tissue of aorta. At the base of the LCC and RCC, the ventricular myocardium of the ostium of the LV comes in direct contact with the aorta while the non-coronary sinus has fibrous tissue at its base because of the fibrous continuity between the aortic and the mitral valves.

\subsection{RVOT vs LVOT sites of origin}

The electrocardiographic distinction between RVOT and LVOT $\mathrm{SOO}$ is insidious because close anatomical structures produce quite similar VA morphologies. Hence, a deep knowledge of the outflow tract anatomy is of paramount importance. From the tricuspid annulus, the RVOT projects in a superior, anterior and leftward direction. The proximal part of RVOT, near to the tricuspid valve, is rightward relative to the aortic root; the main body of RVOT wraps itself around the LVOT and becomes anterior and leftward relative to the aortic root. The RVOT has the following two opposing surfaces: an anterior or "free wall" surface and a posterior or "septal" surface (Fig. 1). Because RVOT and pulmonary valve $(\mathrm{PV})$ are positioned more cranially to the left ventricular counterparts, the posterior (septal) muscular wall of the RVOT borders the right coronary cusp (RCC) and a slight portion of the left coronary cusp (LCC) $[9,10]$.

Anterior structures, close to lead V1, will produce LBBB pattern, while more posterior structures, far from the anterior chest, will produce right bundle branch block (RBBB) pattern. Therefore, RVOT-VAs have usually LBBB owing to the anterior position relative to the LVOT. OT-VAs from the LVOT may have RBBB as well as atypical LBBB morphology depending on the position of the site of origin in the LVOT. For example, RCC is immediately posterior to the septal or posterior RVOT wall so that RCC-VAs have a LBBB pattern. Due to this close anatomical relationship, ECG features of VAs from these two regions are nearly identical and may lead to misdiagnosis of the site of origin. Moving more posteriorly from the RVOT to the lateral mitral annulus produces earlier precordial transition, and the QRS morphology shifts from an LBBB pattern to a RBBB pattern.

When LBBB QRS morphology is observed and the precordial transition occurs at lead V3, it is even more challenging to predict VAs' site of origin because the VAs can originate from both RVOT and LVOT, as demonstrated by Tanner et al. [11]. Several ECG criteria have been proposed to reliably differentiate RVOT from LVOT, such as V2S/V3R index and R/Swave amplitude and duration indexes [12], V2S/V3R index [13], the transitional zone (TZ) index [14] and lead I R-wave amplitude [15]. Unfortunately, these criteria present limitations because the study of the electrical depolarization in the horizontal plane axis is affected by the variable chest-heart relationships, lead position, body habitus and chest wall deformities [16]. The V2 transition ratio overwhelms these limitations by normalizing the VA ECG with respect to the sinus rhythm (SR) ECG. Betensky et al. demonstrated that V2 transition ratio $>0.6$ predicts an LVOT origin with sensitivity at $95 \%$ and specificity at $100 \%$ [17]. 


\section{RVOT FREE WALL}

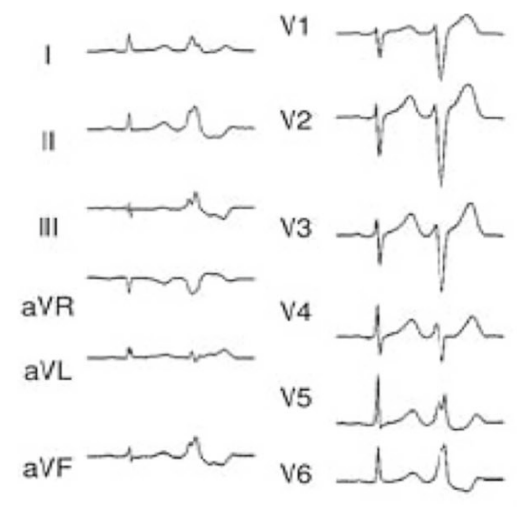

\section{RVOT SEPTUM}
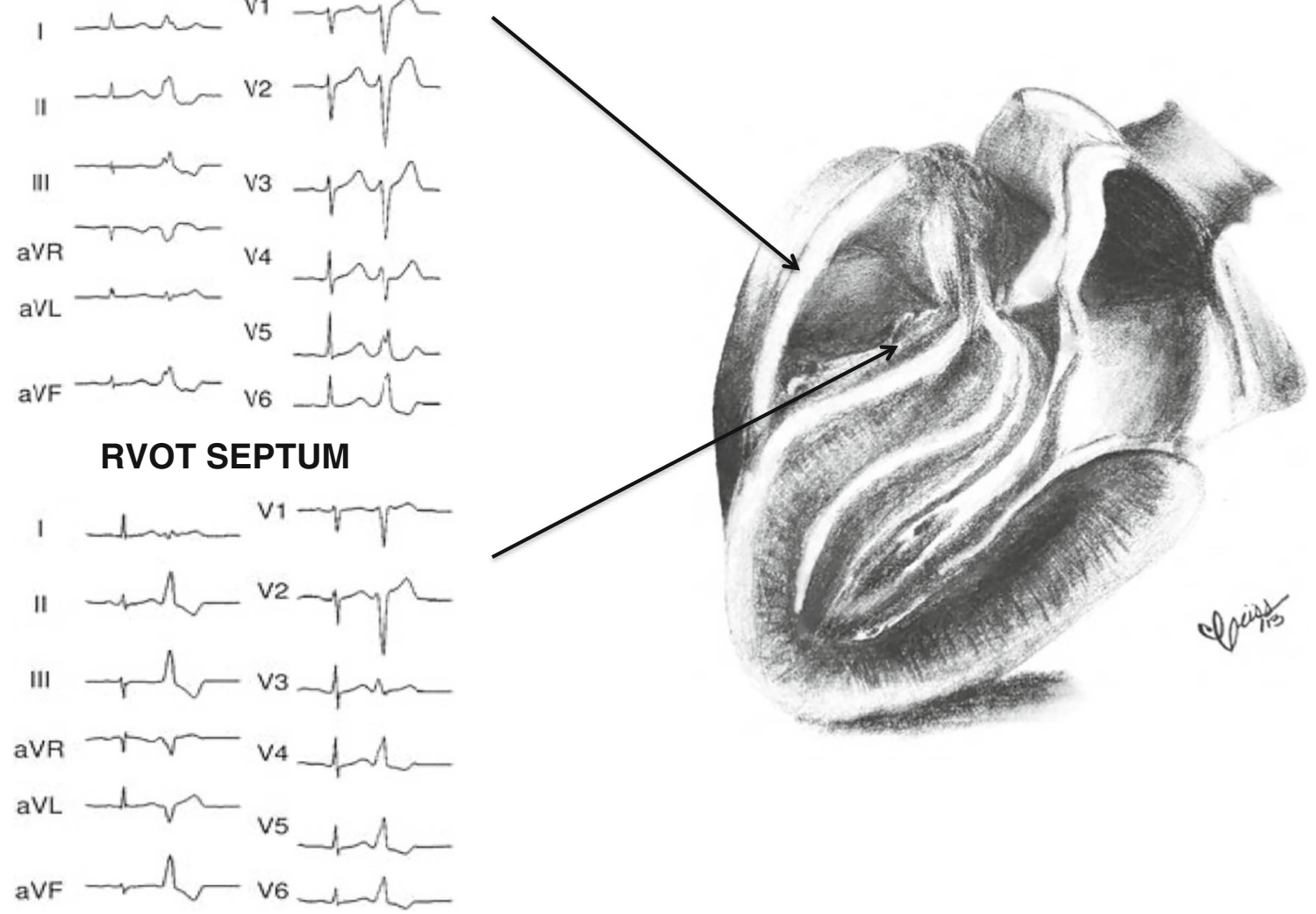

Fig. 1 Twelve-lead ECGs of RVOT-VAs show LBBB and inferior QRS axis. Of note, VAs arising from the RVOT free wall are more anterior and produce later precordial transition rather than those originating from the septal or posterior wall. RVOT, right ventricular outflow tract

More recently, two criteria based on the sagittal axis analysis of heart depolarization have been proposed, which are as follows: the V3R/V7 ratio [18] and the V4/V8 index [19]. The use of right and posterior leads has been demonstrated to provide incremental diagnostic value for differentiating LVOT from RVOT-VAs, regardless cardiac rotation and R/S transition lead, and outperformed the previous ECG algorithms.

\subsection{Right ventricular outflow tract VAs}

The RVOT is the most common SOO, accounting for approximately $80 \%$ of IOT-VAs [8]. In the RVOT, the septum is a more common site of IOT-VA origins than the free wall. Right ventricular outflow tract VAs (RVOT-VAs) have LBBB and inferior QRS axis. Due to its anterior position, close to the sternum and lead V1, usually RVOT-VA shows late precordial transition with $\mathrm{rS}$ or QS wave in lead V1 (Fig. 1). The RVOT free wall is the more anterior surface and will produce VAs with a later precordial transition ( $>$ V4) rather than the RVOT posterior wall, which produces greater anterior vector of activation and an earlier transition (V3 or V4). The free wall VAs demonstrate QRS notching and are often wider (QRS > $140 \mathrm{~ms}$ ) than the posterior wall VAs owning to the not uniform activation of the ventricles; this sequential ventricular depolarization leads to less positive QRS complex in the inferior leads and larger S wave compared to septal VAs [20]. The polarity of lead I helps in distinguishing posterior from anterior septal and free-wall sites [21].

\subsection{Pulmonary artery VAs}

In patients with $\mathrm{LBBB}$ morphology and inferior axis deviation VAs, ablation in RVOT may fail and mapping at the pulmonary sinus cusps (PSCs) should be performed because ventricular myocardium may extend into the pulmonary artery (PA) beyond the semilunar valve and may trigger VAs [20, 22, 23]. The PV is located $1-2 \mathrm{~cm}$ superior from the aortic valve and attaches at the sinotubular junction. Pulmonary artery VAs (PA-VAs) arising from the three pulmonary sinus cusps may display taller $\mathrm{R}$ waves in the inferior leads than RVOT-VAs, though specific ECG characteristics are not well-delineated.

Left pulmonary cusp (LPC), located most superior in the right ventricle, gives rise to VAs with higher inferior lead Rwave amplitudes, a larger aVL/aVR ratio of Q-wave amplitude and a larger R/S ratio in lead V2. VAs arising from right pulmonary cusp (RPC) have a significantly larger R-wave 
amplitude in lead I and longer QRS duration compared with VAs originating from left and anterior pulmonary cusps (APCs). Moreover, the R-wave amplitude in the inferior leads is smaller in VAs from the RPC than LPC but does not differ between VAs from APC and LPC [10, 24].

\subsection{Para-Hisian VAs}

Para-Hisian VAs show relatively narrow LBBB QRS pattern, inferior axis and precordial transition lead $\geq V 3$. The depolarization spreads from the right side of perimembranosus septum, thus producing QS pattern in leads V1-V2 and lower Rwave amplitude in the inferior leads [25].

\subsection{Aortic sinus cusp VAs}

The most common sites of successful ablation in LVOT-VAs are the aortic sinus cusps (ASCs) (Fig. 2). At the base of the LCC and RCC, the ventricular myocardium of the ostium of the LV [26] comes in direct contact with the aorta while the non-coronary cusp (NCC) has fibrous tissue at its base because of the fibrous continuity between the aortic and the mitral valves. This anatomical concept explains how LVOTVAs are rarely associated to NCC.

Due to the rightward tilting of the aortic valve plane in the horizontal plane [10], the LCC is superior, posterior and leftward relative to the other coronary cusps and forms the postero-lateral attachment of the aorta to the LV ostium. The LCC is directly posterior to the RVOT and borders the aortomitral continuity (AMC). The LCC is the most frequent site of origin of LVOT-VAs and is characterized by a RBBB or a multiphasic appearance in lead V1 (an M-/W-shaped pattern), inferior QRS axis on frontal plane and early precordial transition (in leads V1-V2); due to its superior position relative to the other aortic sinus cusps, the LCC-VAs show greater R wave in lead III compared with lead II so that an R-wave amplitude ratio in lead III/II > 0.9 suggests an LCC site of origin [3].

The RCC is the most anterior cusp relative to the sternum, and it lies immediately posterior to the infundibular part of the RVOT. The posterior part of the RCC is adjacent to the central fibrous body, which carries within it the penetrating portion of the His bundle. The RCC-VAs show a LBBB with QS wave in lead I, a precordial transition in leads V2-V3 and greater R wave in lead II than lead III with an R-wave amplitude ratio in lead III/II $<0.9$ [3].

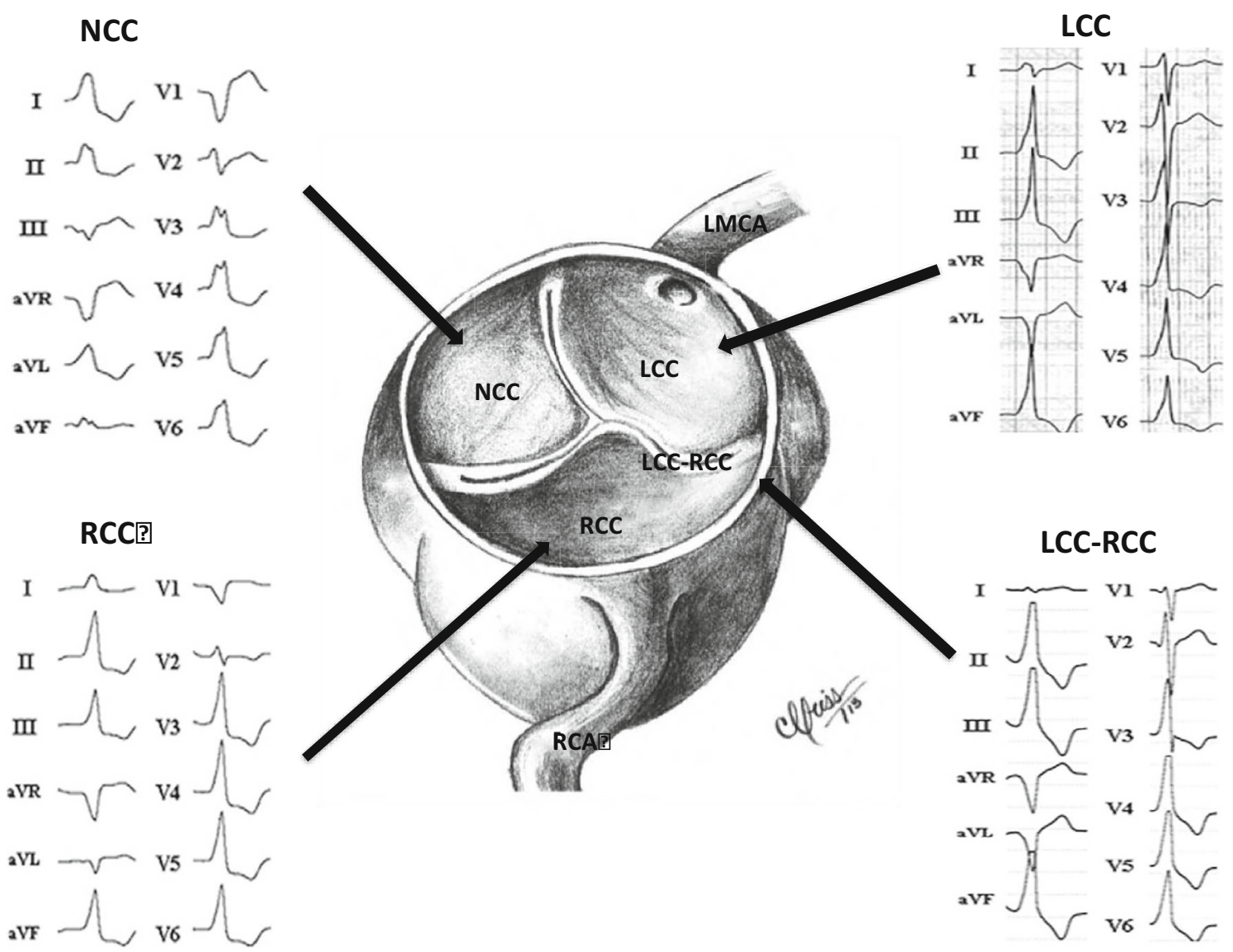

Fig. 2 Twelve-lead ECGs showing VAs originating from the aortic root. Note the different morphologies in leads V1 and V2 with respect of the anatomic origin. NCC, non-coronary cusp; RCC, right coronary cusp; LCC, left coronary cusp; LCC-RCC, left and right coronary cusps commissure 
LVOT-VAs may also originate from the RCC-LCC commissure, and pacing from this site results in LBBB morphology, a qrS wave or a QS wave with notching on the downward deflection in lead V1 and, usually, a precordial transition in lead V3. The early precordial transition and the presence of late potentials in SR at the site of earliest activation are distinguishing features between the RCC-LCC commissure VAs and VAs from the midseptal aspect of the RVOT, which show a notch on downward deflection in lead V1 [27].

The NCC is posterior and rightward relative to the other aortic cusps. Posteriorly, the NCC is in connection with the membranous septum, where it comes in close proximity to the penetrating bundle of His; superiorly, it abuts to interatrial septum so that from the NCC it is possible to record large atrial electrogram and to successfully eliminate atrial tachycardias [28]. The ECG characteristics of NCC-VAs are quite similar to those of RCC-VAs, but, in NCC-VAs, an S wave in lead III with a III/II ratio $<0.65$ and an $\mathrm{R}$ wave in aVL are described [2].

\subsection{Endocardial LVOT-VAs}

LVOT-VAs can also originate from the LV free wall just beneath the aortic annulus or from the superior basal regions of the interventricular septum adjacent to the His bundle region. These VAs usually display an LBBB inferior axis configuration with prominent $\mathrm{R}$ waves in the anterior precordial leads [8].

\subsection{LV summit VAs}

The LV summit (LVS) is the highest portion of LV epicardium, and it is a triangular region bounded by the bifurcation between the left anterior descending (LAD) and the left circumflex (LCx) coronary arteries. It is divided by the great cardiac vein (GCV) in the following 2 regions: a medial and more superior region, inaccessible to the catheter ablation because of close proximity to the coronary arteries and the presence of epicardial fat (the so-called "inaccessible area"); and a more lateral and inferior region presumably suitable for catheter ablation (the "accessible area") [29]. The complex relationship between the LVS and the surrounding anatomical structures makes challenging the approach to VAs arising from this region.

The typical ECG characteristic of LVS-VAs is the slurring of the initial portion of the QRS complex, known as the pseudodelta wave, because the ventricular activation from the epicardial origin requires more time to reach the Purkinje network, resulting in a slow QRS onset during VAs. Several indexes suggest an epicardial site of origin: pseudodelta wave duration $\geq 34 \mathrm{~ms}$, intrinsecoid deflection time (IDT) $\geq 85 \mathrm{~ms}$, QRS duration $>200 \mathrm{~ms}$, RS-wave complex duration > $121 \mathrm{~ms}$, maximum deflection index $(\mathrm{MDI}) \geq 0.55[30,31]$. VAs arising from the accessible area of LV summit have
RBBB pattern, inferior QRS axis, an AVL/aVR Q-wave amplitude ratio 1.1 and an $\mathrm{S}$ wave in leads V5-V6; these arrhythmias may be successfully ablated from the GCV/anterior interventricular vein (AIV). VAs with LBBB pattern have larger $\mathrm{R}$ waves in lead III compared to lead II, later precordial transition (generally after lead V2) and absence of an S wave in leads V5-V6 origin from the "inaccessible area", at the most superior site in the LV [3].

\subsection{Aortomitral continuity VAs}

The AMC is a fibrous connection between the LCC and NCC anteriorly and the anterior leaflet of the mitral valve (MV) posteriorly. The AMC thickens at the both ends to form the left fibrous trigone (that abuts to the posterior aspect of LCC) and the right fibrous trigone (that directly connects NCC with the anterior leaflet of MV). The fibrous trigones are expansions of fibrous tissue, but, inside them, sometimes, it is possible to find myocardial sleeves that may harbour VAs.

$\mathrm{AMC}$ is close to the anterior aspect of the mitral annulus and is just under the LCC so that AMC-VAs may have quite similar morphologies to MV-VAs and LCC-VAs (Fig. 3). Due to its lateral and posterior location, AMC-VAs produce an anteriorly directed ventricular activation resulting in monophasic $\mathrm{R}$ waves with no $\mathrm{S}$ waves in almost all precordial leads. AMC has been divided in the following three parts: anterior, middle and posterior AMC; ECG features have been described for anterior and middle AMC-VAs. Patients with anterior AMC location have LBBB-like QRS pattern, inferior axis and early precordial transition in lead V2, rS in lead V1 and $\mathrm{R}$ in $\mathrm{V} 3$. VAs originating from the mid-AMC show a signature ECG pattern, known as "rebound transition", with $\mathrm{R}$ waves in leads $\mathrm{V} 1$ and $\mathrm{V} 3$ and equal $\mathrm{R}$ and $\mathrm{S}$ waves in $\mathrm{V} 2$. Therefore, an RS pattern in lead V2 is observed in both anterior and mid-AMC origins and $\mathrm{S} / \mathrm{R}$ ratios in leads $\mathrm{V} 1$ and $\mathrm{V} 2$ discriminate between the two sites: these ratios are higher for the anterior AMC location which is close to the sternum and results in low " $r$ " wave. QRS duration and IDT are greater in AMC-VAs than ASC-VAs, and this suggests a subepicardium origin of AMC-VAs [32].

\subsection{Anterior mitral valve VAs}

$\mathrm{MV}$ is located in the posterior portion of the $\mathrm{LV}$ resulting in an early precordial transition that occurs usually in V1 and no later in V2. Moreover, AMV-VAs are characterized by RBBB with a monophasic $\mathrm{R}$ or Rs in leads from $\mathrm{V} 2$ to $\mathrm{V} 6$, no $\mathrm{S}$ wave in V6, inferior axis and negative QRS complex in leads I and aVL. It has been described a late-phase notching of the QRS complex in inferior leads. As AMC-VAs, AMV-VAs show a longer QRS duration and an IDT $\geq 85 \mathrm{~ms}$ owning to the deep annular site of origin [33]. 


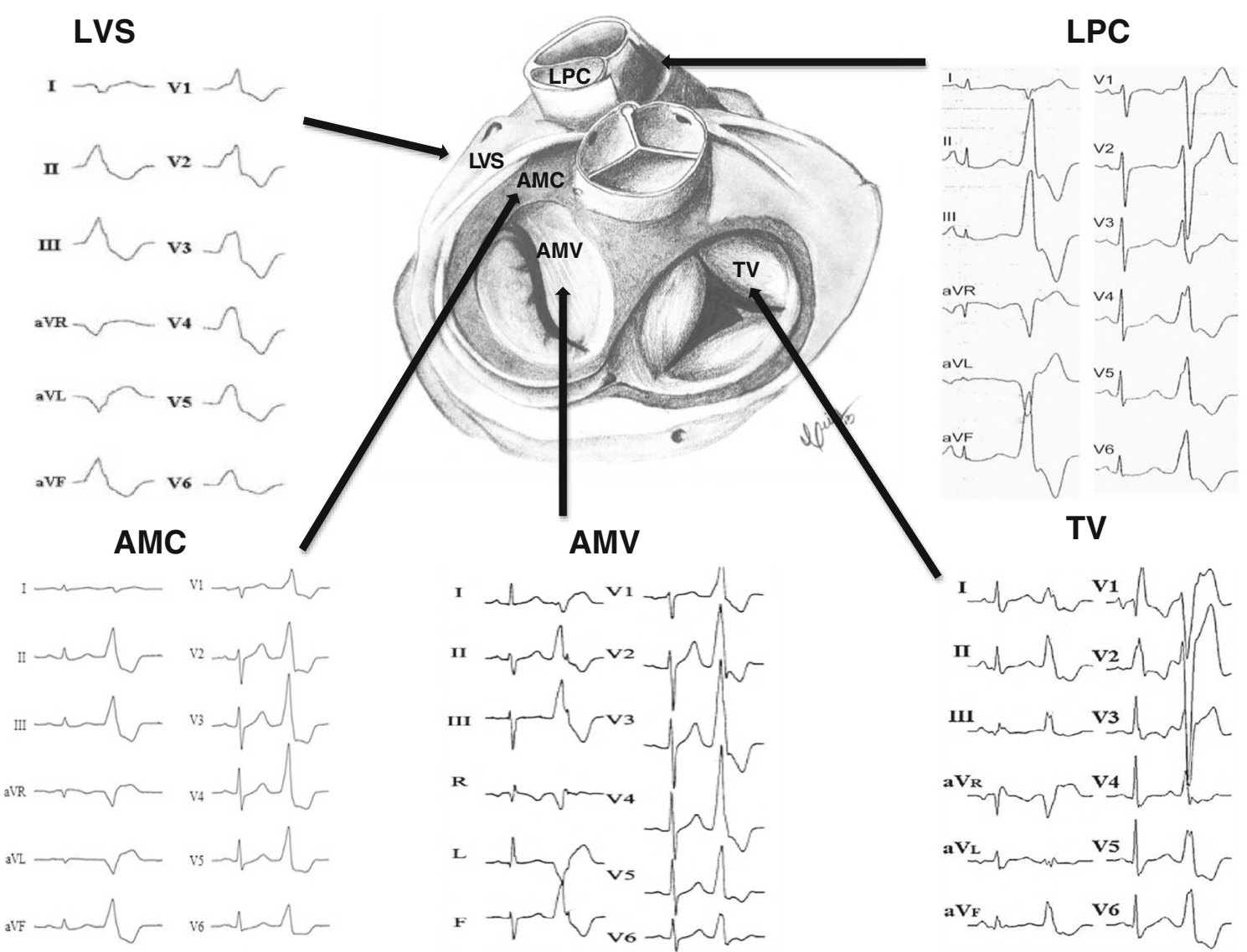

Fig. 3 The morphology of the LVS, AMC, AMV, TV and LPC origins of OT-VAs. Arrhythmias from posterior structures produce earlier precordial transition rather than those originating from anterior structures. LVS, left

\subsection{Intramural focus VAs}

OT-VAs may arise from intramural foci located between the epicardial fat of the LVS and the AMC or in the interventicular septum (IVS) [20]. These arrhythmias display a LBBB QRS pattern and inferior axis, though no specific ECG features have been identified for intramural sites [8].

\section{Management}

Patients presenting with VAs should undergo complete cardiac examination to rule out underlining structural heart disease. If sign of myocardial dysfunction has found, a second-level imaging test, as cardiac magnetic resonance imaging (cMRI), could be necessary to reveal ventricular scarring.

Individuals with frequent asymptomatic PVCs $(\geq 10,000$ $\mathrm{PVCs} / 24 \mathrm{~h}$ ), normal LV ejection fraction and LV dimension should be periodically monitored with echocardiography, along with PVC burden assessment by Holter ECG (class of recommendation IIa) $[8,34]$. Conversely, the presence of arrhythmiainduced cardiomyopathy and/or symptoms requires prompt treatment (Fig. 4). Medical treatment is comprised of beta- ventricular summit; AMC, aorto-mitral continuity; AMV, anterior mitral valve; TV, tricuspid valve; LPC, left pulmonary cusp

blockers, non-dihydropyridine calcium antagonists and class Ic anti-arrhythmic drugs, while class III anti-arrhythmic drugs are not recommended as first-line therapy. If the antiarrhythmic medications are ineffective, not tolerated or not the patient's choice, catheter ablation is recommended for both RVOT-VAs and LVOT-VAs but with different class of recommendation (class of recommendation I for RVOT-VAs and IIa for LVOT-VAs) [8]. Due to the high rate of success with rare complications, catheter ablation is recommended as first-line therapy for symptomatic PVCs from the RVOT. Hence, perioperatively predicting the $\mathrm{SOO}$ with high sensitivity and specificity is of pivotal importance in the management of IOT-VAs.

\section{Mapping and ablation strategy}

Successful ablation of OT-VAs requires detailed electroanatomical mapping superimposed to the anatomic shell obtained with ICE (Fig. 5).

ICE-guided mapping is performed online and shows the ablation catheter location in real time. Usually, ICE probe is placed in the femoral vein and advanced into the RV with the transducer at the base of the RVOT; from this position, ICE 
Fig. 4 Flowchart of management of IOT-VAs. IOT-VAs, idiopathic outflow tract ventricular arrhythmia; PVC, premature ventricular contraction; RVOT, right ventricular outflow tract.

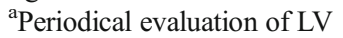
ejection fraction and dimension, ventricular arrhythmia burden with Holter ECG and symptom assessment
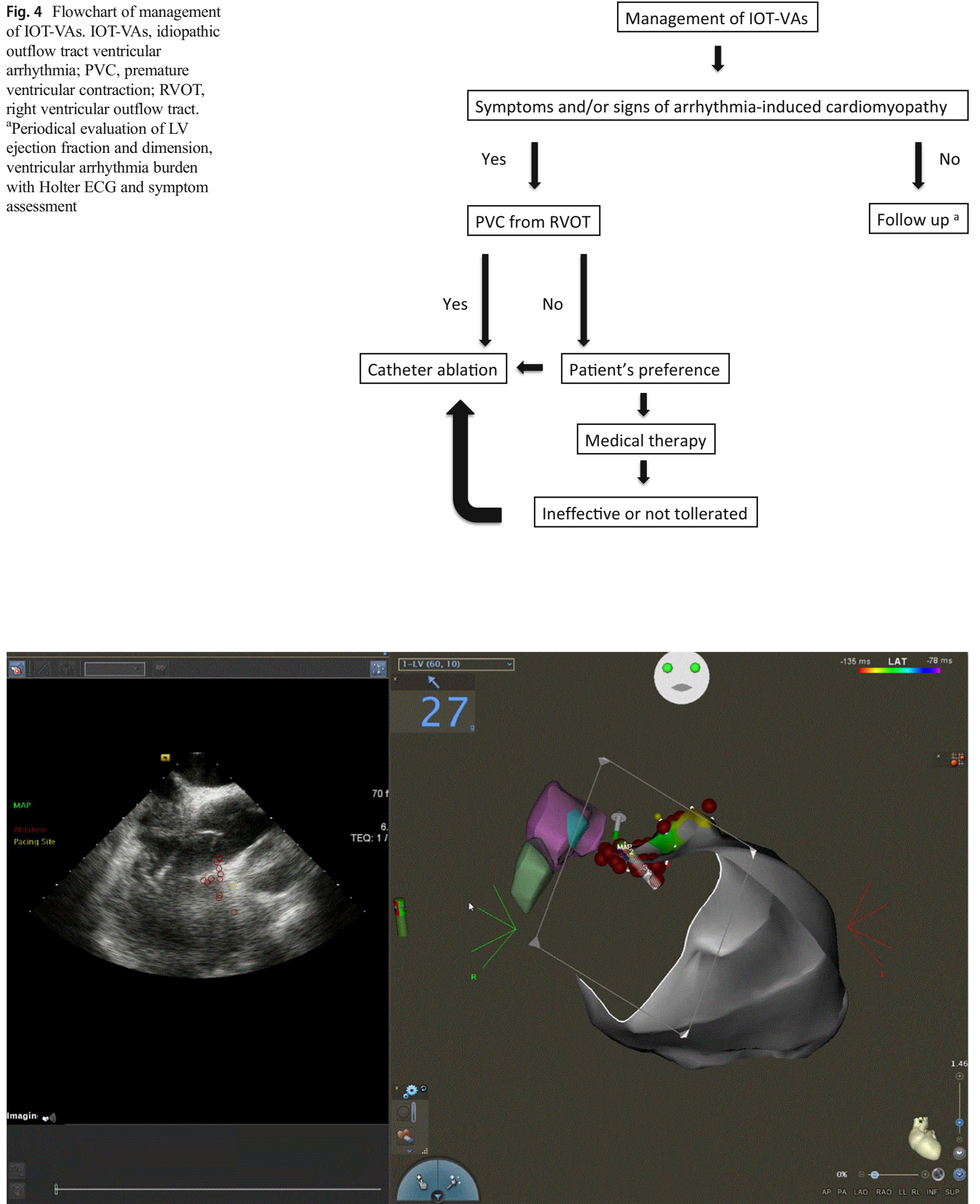

Fig. 5 Electroanatomical mapping and ICE-guiding catheter ablation. On the left, ICE image shows the aortic valve with the cusps and ablation catheter. On the right, electroanatomical map with ICE plane superimposed, showing the shell of left ventricle, aortic valve cusps and catheter ablation. The yellow-green zone represents the site of earliest activation. In both images, the red dots indicate the ablation sets, immediately below the left coronary cusp 
imaging shows LVOT, RVOT, ASCs, coronary arteries, RF lesions and complications [27, 29].

The preferred mapping method is activation mapping that allows to identify the site with earliest presystolic activity, usually consistent with the successful ablation site; this kind of mapping shows the centrifugal activation of the ventricles spreading away from the site of origin, which is identified on the distal bipolar electrodes of a mapping catheter and produces a QS deflection on the distal unipolar recording (Fig. 6). When different contiguous sites show similar earliest activation time, the SOO can be epicardial or intramural. Activation mapping is used for patients with frequent spontaneous ectopies; conversely, in patients with infrequent spontaneous VAs, the induction of arrhythmias is attempted with beta-adrenergic agonists (most frequently isoproterenol) and ventricular burst pacing. When it is impossible to induce ectopic activity, pace mapping can be a useful tool to find the target ablation site, pacing from the source of VA and looking for an identical match (>95\%) between the 12-lead ECG morphologies of spontaneous and the paced OT-VA (Fig. 7). However, pace mapping has low spatial resolution and may be less helpful for aortic root VAs than RVOT-VAs because pacing in the aortic root may not obtain myocardial capture due to the frequent lack of ventricular myocardium within the recesses of the aortic leaflets. Moreover, pacing from the aortic root may not perfectly reproduce the QRS morphology of spontaneous VAs for the presence of preferential conduction, due to the anisotropic conduction between the aortic root site of origin and a relatively distant myocardial breakout site in the RVOT or within the aortic root itself $[35,36]$. Thus, there is a discrepancy between the best pace mapping site and successful ablation site because pace mapping may identify the preferential breakout site but not the VAs' origin [37]. For this reason, the presence of spontaneous VAs at the time of ablation should be maximized withdrawing all anti-arrhythmic drugs at least 5 half-lives before the electrophysiological study (EPS) and using ramifentanyl as anaesthetic drug instead of benzodiazepines and propofol, which can suppress VAs.

Radiofrequency energy is the preferred kind of energy used during catheter ablation procedures, but cryo-thermal ablation may be an alternative to RF ablation in cases with high impedance within the venous system [38], when the VA origin is close to coronary artery [26], to the Bundle of His or is epicardially underneath a fat pad.

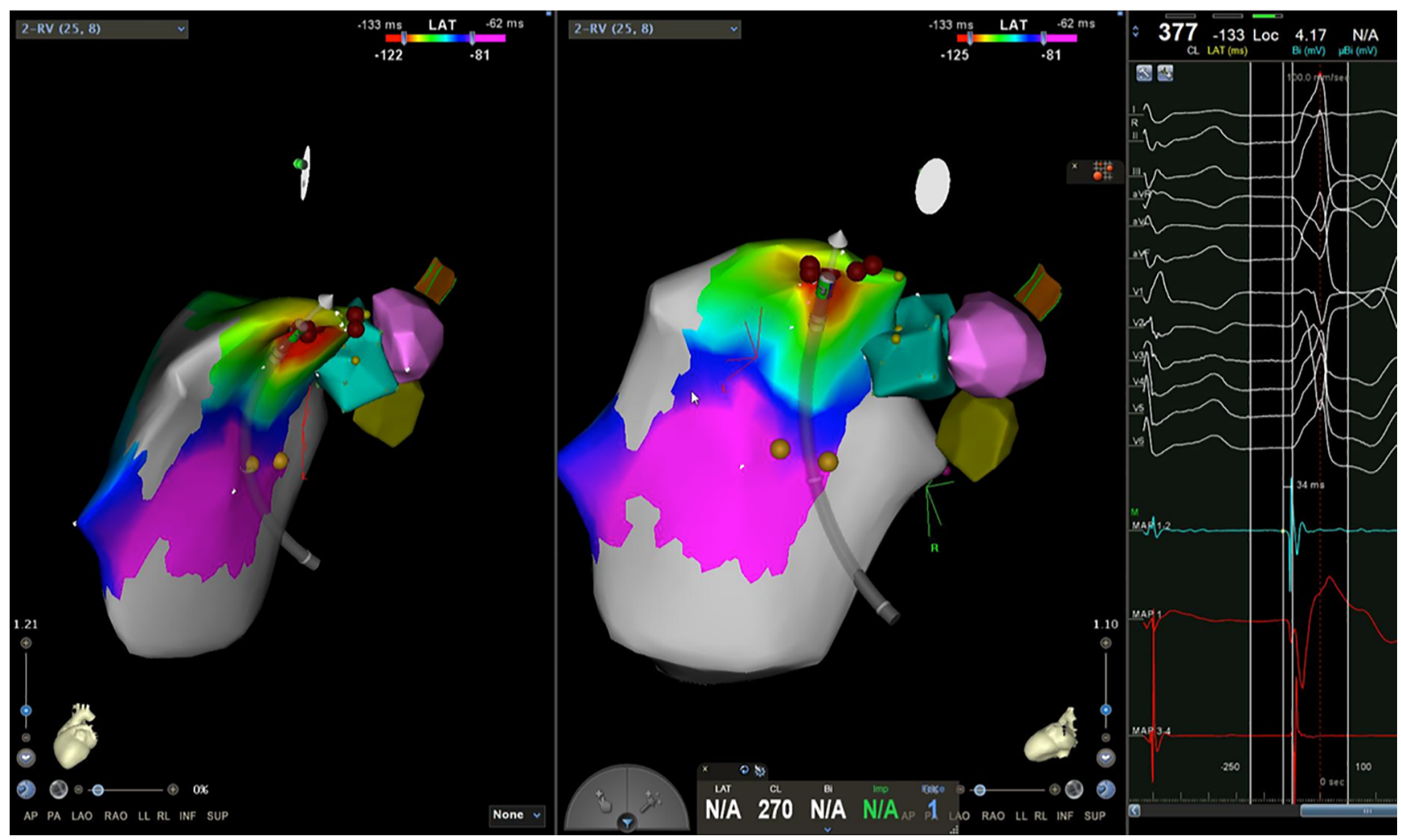

Fig. 6 Activation mapping of PVC from middle septal aspect of RVOT. The shell of right ventricle, aortic cusps and ablation catheter are shown. Activation mapping identifies the earliest site of endocardial activation with red zone; conversely, areas of late activation time are shown by purple. On the right, the interval between the initial deflection on the distal bipolar electrode (MAP 1-2) on the mapping catheter and the beginning of the PVS-QRS on the surface of the ECG represents the presystolic activation time. The unipolar electrode (MAP 1) records a QS deflection meaning that the depolarization wave spreads away from the site of recording. Four ablations (red dots) were delivered at the site of earliest activation with elimination of PVC 


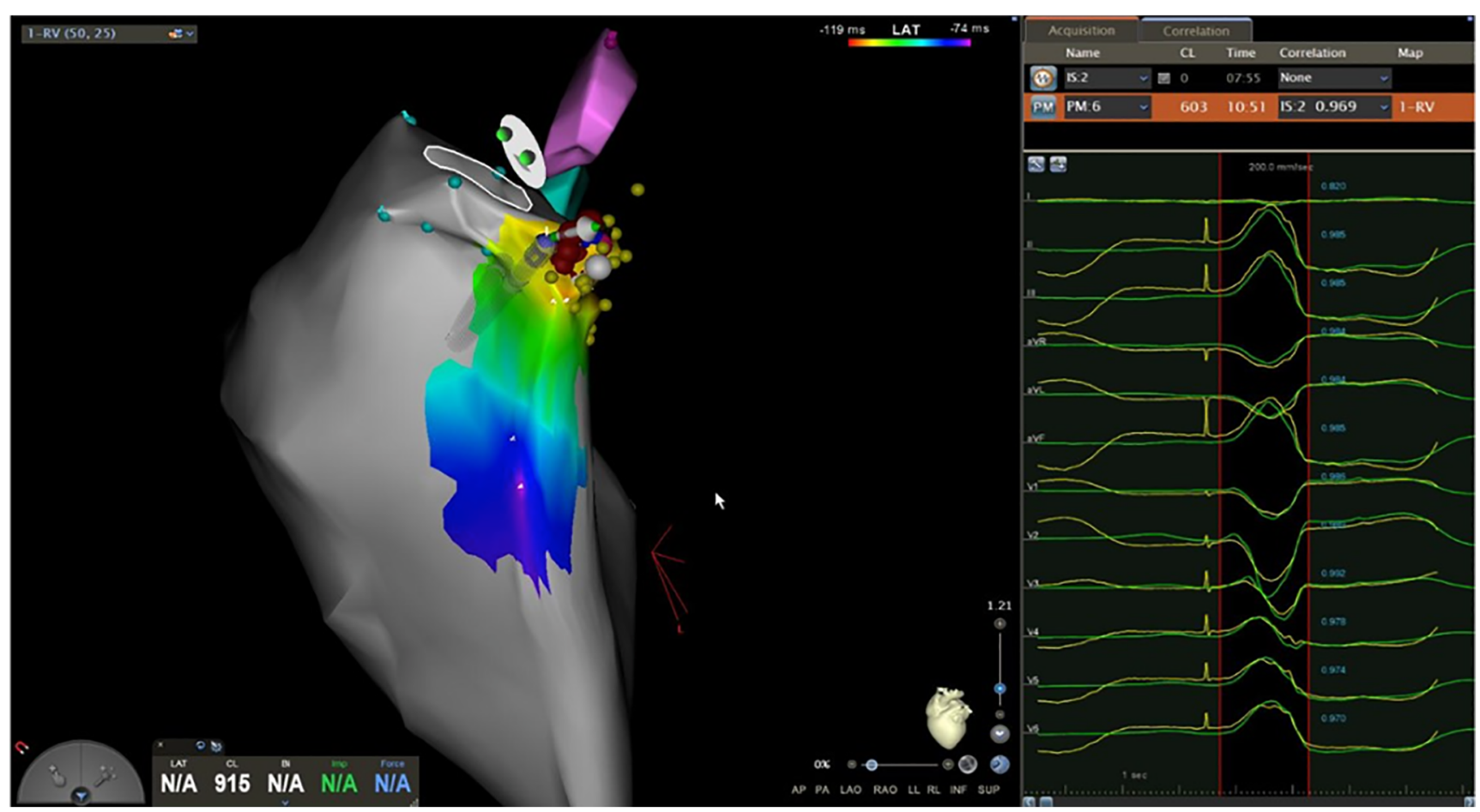

Fig. 7 Pace mapping at the site of earliest activation time. On the left, activation map of RVOT showing a yellow area of early presystolic activation; on the right, pace mapping in the same area resulting in an almost perfect match $(\approx 97 \%)$ between paced complexes and spontaneous PVC

Successful catheter ablation is defined as the absence of spontaneous or inducible VAs with isoproterenol infusion and burst pacing from the RV apex for 30 to 60 min after the last RF delivery.

\section{Challenging sites of origin}

Although ablation of IOT-VAs generally is highly successful with low rates of complications, ablation of VAs originating from LVS and from intramural foci still represents a challenge.

The LVS is located at the most superior, septal and epicardial aspects of LV, in close proximity to coronary arteries. The majority of epicardial LVS-VAs origins at the junction of the GCV/AIV or at the proximal AIV [39], where the earliest activation time is recorded. Coronary angiography is mandatory before radiofrequency catheter ablation (RFCA) to assess the distance between the coronary arteries and the ablation catheter: ablation is not recommended within $5 \mathrm{~mm}$ of a coronary artery and/or within $10 \mathrm{~mm}$ of a coronary ostia [8]. Unfortunately, in approximately $62 \%$ of the patients, the ablation from the coronary venous system (CVS) is not feasible, for the proximity of coronary vasculature or the inability to access the earliest site of activation, or is ineffective for inability to achieve adequate power because of impedance or temperature raise [40]. When ablation from the CVS cannot be pursued, an anatomical approach can be considered, targeting the locations adjacent to the earliest activation site, such as LV endocardium beneath the aortic valve, ASCs and septal RVOT [41, 42]. Shirai et al. [43] reported a success rate of $49 \%$ for anatomical ablation approach in 53 patients with IOT-VAs with the earliest activation time in the CVS. In the same cases, LVS-VAs can be targeted via percutaneous epicardial approach. ECG predictors of successful ablation via direct epicardial approach were described and included a Q-wave ratio $>1.85$ in leads aVL/aVR, absence of $\mathrm{Q}$ wave in $\mathrm{V} 1$ and an $\mathrm{R} / \mathrm{S}$ ratio $>2$ in lead V1 [44]. Likewise ablation from within the CVS, direct epicardial ablation has poor outcomes for the proximity of the coronary arteries and of the phrenic nerve (the position of this nerve should be delineated pacing with $20 \mathrm{~mA}$ to assess for phrenic nerve capture). Overall, LVS-VAs remain a challenge with the lower ablation success rate among IOT-VAs of approximately $67 \%$ [45].

VAs originating from intramural foci in the IVS or in the LVS account for approximately $10 \%$ of LVOT-VAs with LBBB pattern and inferior QRS axis. These arrhythmias do not have peculiar ECG features and often require careful mapping and ablation deliveries in multiple contiguous sites [46]. Yamada [47] suggested that QRS complex that follows < $10 \mathrm{~ms}$ the earliest local ventricular activation on both the endocardial and epicardial sides, unsuccessful RFCA on the side of earliest activation and an only transient suppression of the VA ablating from both endocardium and epicardium should indicate an intramural origin of the VA. Yokokawa [48] described the absence of a matching pace map at the sites of earliest ventricular activation as a clue of septal intramural 


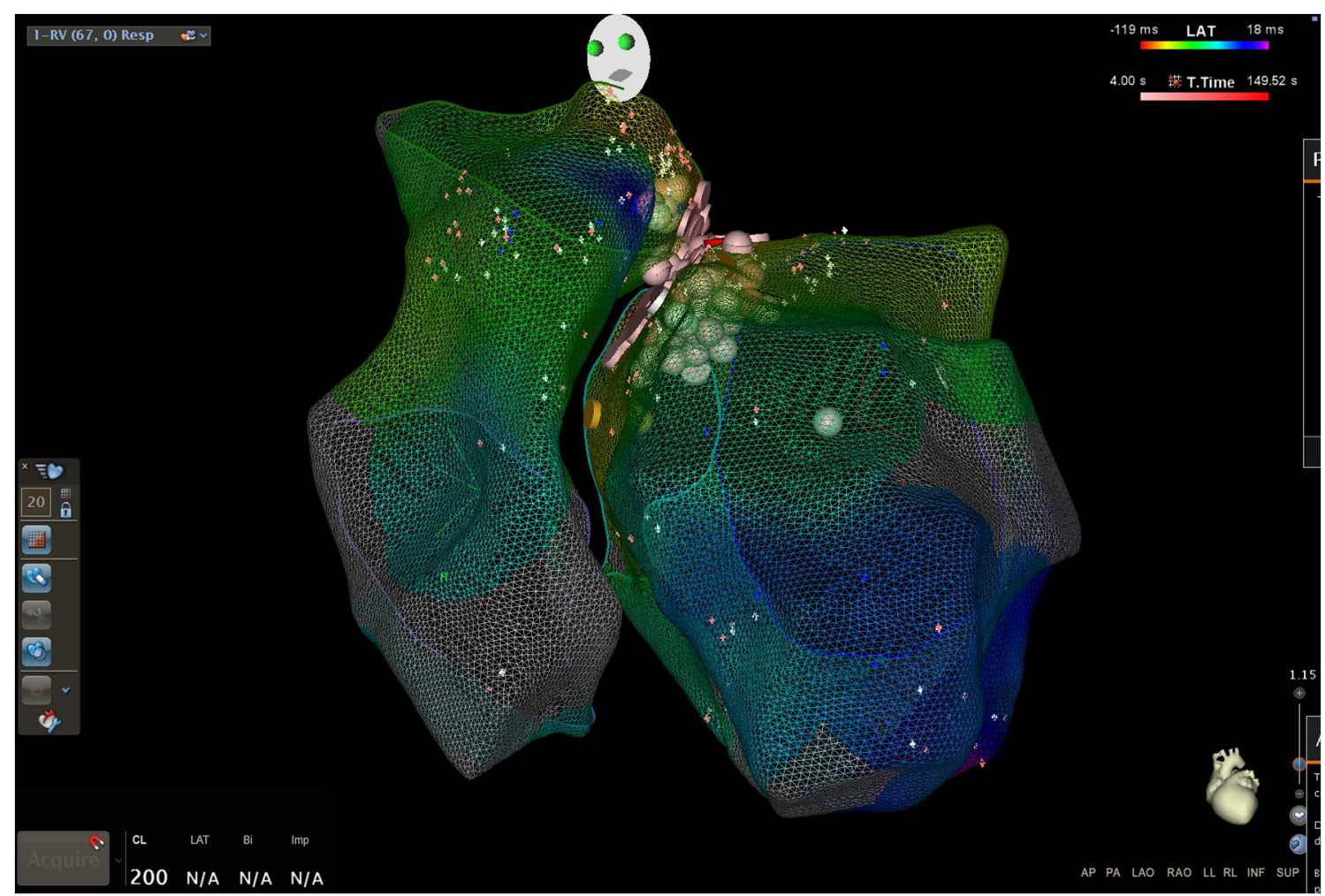

Fig. 8 Left anterior oblique view of electroanatomic reconstruction of both ventricles. The final ablation set for PVC arising from an intramural focus in the basal interventricular septum is shown. Several

focus. Ablation of such arrhythmias is challenging because RF energy could not reach an intramural focus deeply located in the myocardium, accounting for high rates of acute failure and redo procedures. Different techniques for intramural foci have been described, which are as follows: sequential or simultaneous ablation at the sites directly across the wall, the use of infusion-needle catheter ablation [49] or ablation within venous septal perforating branch of the GCV or AIV IVS-VAs [49]. Recently, Di Biase et al. [50] described a case series of 15 patients with multiple sites of equally early activation undergoing sequential RF ablation of intramural foci at all early activation times (Fig. 8). This strategy has been associated with excellent acute- and long-term success (93\%). Anyway, such sequential approach requires thorough activation mapping, also within the CVS, and highly trained operators.

\section{Complications}

Complications are infrequent (approximately $2.4 \%$ of cases) and occur both for LVOT ablations and for RVOT ablations [45]. The most serious complication is coronary artery damage, thrombosis and occlusion [51]. The risk of coronary artery injury is higher for ablation procedures within the aortic root, although it may also occur during ablations of epicardial VAs or VAs originating from the most septal aspect of the radiofrequency energy deliveries (dots), from both the endocardial sides of the septum, can be required to eliminate an arrhythmogenic focus deeply located in the myocardium

RVOT or above the pulmonic valve owning to the close relationship of these structures with the left main coronary artery. During ablation in RCC, RFCA may produce thermal effect on the anterior fat pad that lies between the RVOT and the RCC [52], producing a vagal activation resulting in transient sinus bradycardia followed by transient complete atrioventricular (AV) conduction block. Persistent AV block or RBBB may also occur during ablation of para-Hisian VAs.

Ablation in LVOT can lead to aortic or mitral regurgitation for mechanical trauma or direct energy applications on the valve tissue; also, embolic events can occur during LVOT procedures so that catheter irrigation and periprocedural anticoagulation must be always carried out.

Complications of ablation procedures via the venous system are venous stenosis, venous thrombosis or vein rupture with pericardial effusion followed by cardiac tamponade [53]; therefore, pericardiocentesis should always be preoperatively planned [54].

\section{Conclusions}

IOT-VAs represent an intriguing clinical challenge due to the protean electrocardiographic features resulting from the heterogeneous sites of origin. Therefore, pinpointing the source of the VA before the ablation procedure may be difficult. The 
interpretation of the 12-lead ECG morphology of the patient's VA may facilitate SOO localization allowing to perform RFCA procedures in a safer fashion. Electroanatomical mapping and ICE are of pivotal importance for ablation success. VAs originating from LVS and from intramural foci remain the challenge in electrophysiology, and, though different strategies have been developed to improve outcomes and safety of ablation procedures, new studies are eagerly awaited to address this issue.

\section{Compliance with ethical standards}

Conflict of interest The authors declare that they have no conflict of interest.

This research did not receive any specific grant from founding agencies in the public, commercial or not-for-profit sectors.

\section{References}

1. Kim RJ, Iwai S, Markowitz SM, Shah BK, Stein KM, Lerman BB. Clinical and electrophysiological spectrum of idiopathic ventricular outflow tract arrhythmias. J Am Coll Cardiol. 2007;49(20):203543.

2. Yamada T. Idiopathic ventricular arrhythmias: relevance to the anatomy, diagnosis and treatment. J Cardiol. 2016;68(6):463-71.

3. Kumagai K. Idiopathic ventricular arrhythmias arising from the left ventricular outflow tract: tips and tricks. J Arrhythmia. 2014;30(4): 211-21.

4. Yamada T, Doppalapudi H, McElderry HT, Okada T, Murakami Y, Inden $\mathrm{Y}$, et al. Idiopathic ventricular arrhythmias originating from the papillary muscles in the left ventricle: prevalence, electrocardiographic and electrophysiological characteristics, and results of the radiofrequency catheter ablation. J Cardiovasc Electrophysiol. 2010;21:62-9.

5. Crawford T, Mueller G, Good E, Jongnarangsin K, Chugh A, Pelosi $\mathrm{F} \mathrm{Jr}$, et al. Ventricular arrhythmias originating from papillary muscles in the right ventricle. Heart Rhythm. 2010;7(6):725-30.

6. Sadek MM, Benhayon D, Sureddi R, Chik WM, Santangeli P, Supple GE, et al. Idiopathic ventricular arrhythmias originating from the moderator band: electrocardiographic characteristics and treatment by catheter ablation. Heart Rhythm. 2015;12(1):67-75.

7. Dukkipati SR, Choudry S, Koruth JS, Miller MA, Whang W, Reddy VY. Catheter ablation of ventricular tachycardia in structurally normal hearts indications, strategies, and outcomes - part I. J Am Coll Cardiol. 2017;70:2909-23.

8. Cronin EM, Bogun FM, Maury P, Peichl P, Chen M, Namboodiri $\mathrm{N}$, et al. $2019 \mathrm{HRS} / \mathrm{EHRA} / \mathrm{APHRS} / \mathrm{LAHRS}$ expert consensus statement on catheter ablation of ventricular arrhythmias. Europace. 2019;00:1-147.

9. Ho SY. Structure and anatomy of the aortic root. Eur J Echocardiogr. 2009;10(1):4-11.

10. Hutchinson MD, Garcia FC. An organized approach to the localization, mapping, and ablation of outflow tract ventricular arrhythmias. J Cardiovasc Electrophysiol. 2013;24(10):1189-97.

11. Tanner H, Hindricks G, Schirdewahn P, Kobza R, Dorszewski A, Piorkowski C, et al. Outflow tract tachycardia with $\mathrm{R} / \mathrm{S}$ transition in lead V3. Six different anatomic approaches for successful ablation. J Am Coll Cardiol. 2005;45:418-23.

12. Ouyang F, Fotuhi P, Ho SY, Hebe J, Volkmer M, Goya M, et al. Repetitive monomorphic ventricular tachycardia originating from the aortic sinus cusp. J Am Coll Cardiol. 2002;39:500-8.
13. Yoshida N, Yamada T, McElderry HT, Inden Y, Shimano M, Murohara T, et al. A novel electrocardiographic criterion for differentiating a left from right ventricular outflow tract tachycardia origin: the V2s/V3R index. J Cardiovasc Electrophysiol. 2014;25(7): $747-53$.

14. Yoshida N, Inden Y, Uchikawa T, Kamiya H, Kitamura K, Shimano $\mathrm{M}$, et al. Novel transitional zone index allows more accurate differentiation between idiopathic right ventricular outflow tract and aortic sinus cusp ventricular arrhythmias. Heart Rhythm. 2011;8:34956.

15. Xie S, Kubala M, Liang JJ, Hayashi T, Park J, Padros IL, et al. Lead I R-wave amplitude to differentiate idiopathic ventricular arrhythmias with left bundle branch block right inferior axis originating from the left versus right ventricular outflow tract. J Cardiovasc Electrophysiol. 2018;29:1515-22.

16. Anter E, Frankel DS, Marchlinski FE, Dixit S. Effect of electrocardiographic lead placement on localization of outflow tract tachycardias. Heart Rhythm. 2012;9:697-703.

17. Betensky BP, Park RE, Marchlinski FE, Hutchinson MD, Garcia FC, Dixit S, et al. The V2 transition ratio: a new electrocardiographic criterion for distinguishing left from right ventricular outflow tract tachycardia origin. J Am Coll Cardiol. 2011;57(22):2255-62.

18. Cheng D, Ju W, Zhu L, Chen K, Zhang F, Chen H, et al. V3R/V7 index. A novel electrocardiographic criterion for differentiating left from right ventricular outflow tract arrhythmias origins. Circ Arrhythm Electrophysiol. 2018;11(11) e006243.

19. Zhang F, Hamon D, Fang Z, Xu Y, Yang B, Ju W, et al. Value of a posterior electrocardiographic lead for localization of ventricular outflow tract arrhythmias. The V4/V8 ratio. J Am Coll Clin Electrophysiol. 2017;3(7):678-86.

20. Lerman BB. Mechanism, diagnosis, and treatment of outflow tract tachycardia. Nat Rev Cardiol. 2015;12(10):597-608.

21. Dixit S, Gerstenfeld EP, Callans DJ, Marchlinski FE. Electrocardiographic patterns of superior right ventricular outflow tract tachycardias: distinguishing septal and free-wall sites of origin. J Cardiovasc Electrophysiol. 2003;14:1-7.

22. Liu CF, Cheung JW, Thomas G, Ip JE, Markowitz SM, Lerman BB. Ubiquitous myocardial extensions into the pulmonary artery demonstrated by integrated intracardiac echocardiography and electroanatomic mapping changing the paradigm of idiopathic right ventricular outflow tract arrhythmias. Circ Arrhythm Electrophysiol. 2014;7(4):691-700.

23. Hasdemir C, Aktas S, Govsa F, Aktas EO, Kicak A, Bozkaya YT, et al. Demonstration of ventricular myocardial extensions into the pulmonary artery and aorta beyond the ventriculo-arterial junction. PACE. 2007;30(4):534-9.

24. Liao Z, Zhan X, Wu S, Xue Y, Fang X, Liao H, et al. Idiopathic ventricular arrhythmias originating from the pulmonary sinus cusp prevalence, electrocardiographic/electrophysiological characteristics, and catheter ablation. J Am Coll Cardiol. 2015;66(23):263344.

25. Yamauchi Y, Aonuma K, Takahashi A, Sekiguchi Y, Hachiya H, Yokoyama Y, et al. Electrocardiographic characteristics of repetitive monomorphic right ventricular tachycardia originating near the Hisbundle. J Cardiovasc Electrophysiol. 2005;16:1041-8.

26. Yamada T, Litovsky SH, Kay GN. The left ventricular ostium: an anatomic concept relevant to idiopathic ventricular arrhythmias. Circ Arrhythm Electrophysiol. 2008;1(5):396-404.

27. Bala R, Garcia FC, Hutchinson MD, Gesternfeld EP, Dhruvakumar $\mathrm{S}$, Dixit $\mathrm{S}$, et al. Electrocardiographic and electrophysiologic features of ventricular arrhythmias originating from the right/left coronary cusp commissure. Heart Rhythm. 2010;7(3):312-22.

28. Lin D, Ilkhanoff L, Gerstenfeld E, Dixit S, Beldner S, Bala R, et al. Twelve-lead electrocardiographic characteristics of the aortic cusp region guided by intracardiac echocardiography and electroanatomic mapping. Heart Rhythm. 2008;5(5):663-9. 
29. Enriquez A, Malavassi F, Saenz LC, Supple G, Santangeli P, Marchilinski FE, et al. How to map and ablate left ventricular summit arrhythmias. Heart Rhythm. 2017;14(1):141-8.

30. Berruezo A, Mont L, Nava S, Chueca E, Bartholomay E, Brugada J. Electrocardiographic recognition of the epicardial origin of ventricular tachycardias. Circulation. 2004;109(15):1842-7.

31. Daniels DV, Lu YY, Morton JB, Santucci PA, Akar JG, Green A, et al. Idiopathic epicardial left ventricular tachycardia originating remote from the sinus of Valsalva: electrophysiological characteristics, catheter ablation, and identification from the 12-lead electrocardiogram. Circulation. 2006;113:1659-66.

32. Chen J, Hoff PI, Rossvoll O, De Bortoli A, Solheim E, Sun L, et al. Ventricular arrhythmias originating from the aortomitral continuity: an uncommon variant of left ventricular outflow tract tachycardia. Europace. 2012;14:388-95.

33. Tada H, Ito S, Naito S, Kurosaki K, Kubota S, Sugiyasu A, et al. Idiopathic ventricular arrhythmia arising from the mitral annulus: a distinct subgroup of idiopathic ventricular arrhythmias. J Am Coll Cardiol. 2005;45:877-86.

34. Niwano SY, Wakisaka H, Niwano H, Fukaya H, Kurokawa S, Kiryu M, et al. Prognostic significance of frequent premature ventricular contractions originating from the ventricular outflow tract in patients with normal left ventricular function. Heart. 2009;95: 1230-7.

35. Yamada T, Platonov M, McElderry HT, Kay GN. Left ventricular outflow tract tachycardia with preferential conduction and multiple exits. Circ Arrhythm Electrophysiol. 2008;1:140-2.

36. Yamada T, Murakami Y, Yoshida N, Okada T, Shimizu T, Toyama $\mathrm{J}$, et al. Preferential conduction across the ventricular outflow septum in ventricular arrhythmias originating from the aortic sinus cusp. J Am Coll Cardiol. 2007;50(9):884-91.

37. Lavalle C, Mariani MV, Della Rocca DG, Natale A. Role of surface electrocardiogram in the era of high-resolution mapping and imaging systems - back to the future. J Thorac Dis. 2019;11(Suppl 3): S307-10.

38. Aoyama H, Nakagawa H, Pitha JV, Khammar GS, Chandrasekaran $\mathrm{K}$, Matsudaira K, et al. Comparison of cryothermia and radiofrequency current in safety and efficacy of catheter ablation within the canine coronary sinus close to the left circumflex coronary artery. J Cardiovasc Electrophysiol. 2005;16:1218-26.

39. Rivera S, Vecchio N, Ricapito P, Ayala-Paredes F. Nonfluoroscopic catheter ablation of arrhythmias with origin at the summit of the left ventricle. J Interv Card Electrophysiol. 2019. https://doi.org/10.1007/s10840-019-00522-1.

40. Mountantonakis SE, Frankel DS, Tschabrunn CM, Hutchinson MD, Riley MP, Lin D, et al. Ventricular arrhythmias from the coronary venous system: prevalence, mapping and ablation. Heart Rhythm. 2015;12(6):1145-53.

41. Frankel DS, Mountantonakis SE, Dahu MI, Marchlinski FE. Elimination of ventricular arrhythmias originating from the anterior interventricular vein with ablation in the right ventricular outflow tract. Circ Arrhythm Electrophysiol. 2014;7:984-5.

42. Nagashima K, Choi EK, Lin KY, Kumar S, Tedrow UB, Koplan $\mathrm{BA}$, et al. Ventricular arrhythmias near the distal great cardiac vein challenging arrhythmia for ablation. Circ Arrhythm Electrophysiol. 2014:7:906-12.

43. Shirai Y, Santangeli P, Liang JJ, Garcia FC, Supple GE, Frankel DS, et al. Anatomical proximity dictates successful ablation from adjacent sites for outflow tract ventricular arrhythmias linked to the coronary venous system. Europace. 2019;21(3):484-91.

44. Santangeli P, Marchlinski FE, Zado ES, Benhayon D, Hutchinson MD, Lin D, et al. Percutaneous epicardial ablation of ventricular arrhythmias arising from the left ventricular summit: outcomes and eclectrocardiogram correlates of success. Circ Arrhythm Electrophysiol. 2015;8:337-43.

45. Latchamsetty R, Yokokawa M, Morady F, Kim HM, Mathew S, Tilz R, et al. Multicenter outcomes for catheter ablation of idiopathic premature ventricular complexes. J Am Coll Cardiol EP. 2015;1: 116-23.

46. Gianni C, Mohanty S, Trivedi C, Di Biase L, Al-Ahmad A, Natale A, et al. Alternative approaches for ablation of resistant ventricular tachycardia. Card Electrophysiol Clin. 2017;9(1):93-8.

47. Yamada T, Maddox WR, McElderry HT, Doppalapudi H, Plumb VJ, Kay GN. Radiofrequency catheter ablation of idiopathic ventricular arrhythmias originating from intramural foci in the left ventricular outflow tract: efficacy of sequential versus simultaneous unipolar catheter ablation. Circ Arrhythm Electrophysiol. 2015;8: 344-52.

48. Yokokawa M, Good E, Chugh A, Pelosi F, Crawford T, Jongnarangsin $\mathrm{K}$, et al. Intramural idiopathic ventricular arrhythmias originating in the intraventricular septum. Circ Arrhythm Electrophysiol. 2012;5:258-63.

49. Sapp JL, Beeckler C, Pike R, Parkash R, Gray CJ, Zeppenfeld $\mathrm{K}$, et al. Initial human feasibility of infusion needle catheter ablation for refractory ventricular tachycardia. Circulation. 2013;128:2289-95.

50. Di Biase L, Romero J, Zado ES, Diaz JC, Gianni C, Hranitzki PM, et al. Variant of ventricular outflow tract ventricular arrhythmias requiring ablation from multiple sites: intramural origin. Heart Rhythm. 2019;16(5):724-32.

51. Pons M, Beck L, Leclercq F, Ferriere M, Albat B, Davy JM. Chronic left main coronary artery occlusion: a complication of radiofrequency ablation of idiopathic left ventricular tachycardia. PACE. 1997;20:1874-6.

52. Yamada T, Yoshida Y, Inden Y, Murohara T, Kay GN. Vagal reflex provoked by radiofrequency catheter ablation in the right aortic sinus cusp: a Bezold-Jarisch-like phenomenon. J Interv Card Electrophysiol. 2008;23:199-204.

53. Obel OA, d'Avila A, Neuzil P, Saad EB, Ruskin JN, Reddy VY. Ablation of left ventricular epicardial outflow tract tachycardia from the distal great cardiac vein. J Am Coll Cardiol. 2006;48:1813-7.

54. Mariani MV, Gatto MC, Piro A, Fedele F, Lavalle C. Delayed efficacy of radiofrequency catheter ablation on arrhythmias originating in the interventricular basal septum. Clin Case Rep. 2019:1-6.

Publisher's note Springer Nature remains neutral with regard to jurisdictional claims in published maps and institutional affiliations. 